\title{
INTERPOLATION BY MULTIPLIERS ON CERTAIN SPACES OF ANALYTIC FUNCTIONS
}

\author{
K. MOSALEHEH and K. SEDDIGHI
}

(Received 20 March 1998)

\begin{abstract}
Let $\mathscr{H}$ be a Hilbert space of analytic functions on a planar domain $G$ such that, for each $\lambda$ in $G$, the linear functional $e_{\lambda}$ of evaluation at $\lambda$ is bounded on $\mathscr{H}$. Furthermore, assume that $z \mathscr{H} \subset \mathscr{H}$ and $\sigma\left(M_{z}\right)=\bar{G}$ is an $M$-spectral set for $M_{z}$, the operator of multiplication by $z$. This paper is devoted to the study of interpolation by multipliers of the space $\mathscr{H}$ and, in particular, the Dirichlet space.
\end{abstract}

Keywords and phrases. Interpolation, multipliers, Dirichlet space, finitely connected domains.

2000 Mathematics Subject Classification. Primary 47B38; Secondary 46E20.

1. Introduction. Let $G$ be a finitely connected domain in the complex plane $\mathbb{C}$. The Bergman norm of a function $f$ analytic on $G$ is defined by

$$
\|f\|_{2}^{2}=\int_{G}|f|^{2} d A,
$$

where $d A$ denotes the usual Lebesgue area measure. The Bergman space $L_{a}^{2}(G)$ is the set of all functions analytic on $G$ such that $\|f\|_{2}<\infty$.

The Dirichlet space $D(G)$ is the Hilbert space of analytic functions on $G$ whose derivative lies in $L_{a}^{2}(G)$. The Dirichlet norm is defined by

$$
\|f\|_{D}^{2}=|f(\omega)|^{2}+\int_{G}\left|f^{\prime}\right|^{2} d A
$$

for $f \in D(G)$ and a fixed point $\omega$ in $G$. This $\omega$ is called the base point of $D(G)$. It is shown by Chan [5] that the norms obtained by fixing different points are equivalent. If $G=U$ is the open unit disc and $f(z)=\sum_{n=0}^{\infty} a_{n} z^{n}$, then $\|f\|_{D}^{2}=\left|a_{0}\right|^{2}+\pi \sum_{n=1}^{\infty} n\left|a_{n}\right|^{2}$. If $f \in D(U)$, then $\sum_{n=1}^{\infty}\left|a_{n}\right|^{2}<\infty$, and so $f \in H^{2}$. Therefore, $D(U) \subset H^{2}$.

An analytic function $\varphi$ in $G$ is said to be a multiplier for $D(G)$ if $\varphi f \in D(G)$ for all $f \in D(G)$. The set of all multipliers of the Dirichlet space is denoted by $M(D(G))$. For each $\varphi \in M(D(G))$, define the multiplication operator $M_{\varphi}: D(G) \rightarrow D(G)$ by $M_{\varphi} f=\varphi f$ for all $f \in D(G)$. Clearly, $M_{\varphi}$ is a bounded operator on $D(G)$. The norm of $M_{\varphi}$ is the usual norm. If we identify $\varphi$ with $M_{\varphi}$, then $M(D(G))$ can be thought of as a subspace of the space of bounded linear operators on $D(G)$.

A sequence $\left(\omega_{n}\right)$ in $G$ is said to be an interpolating sequence for $M(D(G))$ if, for each bounded sequence $\left(a_{n}\right) \subset \mathbb{C}$, there exists $\varphi \in M(D(G))$ such that $\varphi\left(\omega_{n}\right)=a_{n}$.

Let $G_{1}$ and $G_{2}$ be two conformally equivalent regions and let $h: G_{1} \rightarrow G_{2}$ be a conformal map which takes the base point that defines the norm of $D\left(G_{1}\right)$ to that of $D\left(G_{2}\right)$. Now, since $\int_{G_{2}}\left|f^{\prime}\right|^{2} d A=\int_{G_{1}}\left|(f \circ h)^{\prime}\right|^{2} d A$, the composition operator $C_{h}$ from $D\left(G_{2}\right)$ 
onto $D\left(G_{1}\right)$ is an isometry. Therefore, $D\left(G_{1}\right)$ is unitarily equivalent to $D\left(G_{2}\right)$. We also observe that the map $M\left(D\left(G_{2}\right)\right) \rightarrow M\left(D\left(G_{1}\right)\right)$ given by $\varphi \rightarrow \varphi \circ h$ is an isomorphism.

A circular domain is a domain that is obtained by removing a finite number of disjoint closed subdisks from the open unit disk. We can assume that each removed closed subdisk has a positive radius since otherwise that point is a removable singularity of every function in $D(G)$. We only prove this for the origin. If $f \in D(G)$, where $G$ contains $B(0, R) \backslash\{0\}$, then $f(z)=\sum_{n=-\infty}^{\infty} a_{n} z^{n}, r<|z|<R$ for some $0<r<R$. We have $\pi \sum_{n \neq 0} n\left|a_{n}\right|^{2}\left(R^{2 n}-r^{2 n}\right)=\int_{r<|z|<R}\left|f^{\prime}\right|^{2} d A<\int_{G}\left|f^{\prime}\right|^{2} d A$. Now, if $r \rightarrow 0$, then $a_{n}=0$ for $n<0$. Therefore, $f$ is analytic at 0 .

If $G$ is a finitely connected domain in $\mathbb{C}$, there exists a conformal map $\psi$ of $G$ onto some circular domain $\Omega$, and so $D(G)$ is isometric to $D(\Omega)$. If $G$ is a finitely connected domain in $\mathbb{C}$ and $K_{1}, K_{2}, \ldots, K_{N}$ are the bounded components of $\mathbb{C} \backslash G$, then $G_{0}=G \cup$ $K_{1} \cup K_{2} \cup \cdots \cup K_{N}$ and $G_{i}=(\mathbb{C} \cup\{\infty\}) \backslash K_{i}, 1 \leq i \leq N$, are simply connected regions. By Cauchy integral formula, every function $f$ analytic on $G$ can be written as a sum $f=f_{0}+f_{1}+\cdots+f_{N}$, where each $f_{i}$ is analytic on $G_{i}$ and $f_{k}(\infty)=0$ if $k \neq 0$. Moreover, this summation is unique up to the order.

We use the notation $H(G)$ for the algebra of all analytic functions in $G$ and $H^{\infty}(G)$ for the algebra of all bounded analytic functions in $G$. If $G$ is unbounded, $H_{0}(G)$ is the space of all analytic functions in $G$ that vanish at infinity. We say that $M(D(G))$ is rotation invariant if whenever $h \in M(D(G)), h_{\theta} \in M(D(G))$, where $h_{\theta}(z)=h\left(e^{-i \theta} z\right)$.

Axler [1] considered the interpolation sequence for $M(D(U))$. In this paper, we consider the interpolation sequence for $M(D(G))$. Which we treat in Section 2. In Section 3, we consider the interpolation sequence for $M(H)$, where $H$ is a Hilbert space of analytic functions on $G$ and $M(H)$ is the set of all multipliers of $H$.

2. Interpolation sequence for $M(D(G))$. We use Rosenthal-Dor theorem as proved by Rosenthal [11] and Dor [9].

THEOREM 2.1 (Rosenthal-Dor theorem). Suppose that $E$ is a Banach space and $\left(e_{n}\right)$ is a bounded sequence in $E$. Then there exists a subsequence $\left(e_{n_{k}}\right)$ such that either

(i) the map $\left(a_{k}\right) \mapsto \sum_{k=1}^{\infty} a_{k} e_{n_{k}}$ is an isomorphism of $l^{1}$ into E; or

(ii) $\lim _{k \rightarrow \infty} \varphi\left(e_{n_{k}}\right)$ exists for every $\varphi \in E^{*}$.

As we see in Rosenthal-Dor theorem, we must work with a Banach space. Therefore, we need to make $M(D(\Omega))$, where $\Omega$ is a circular domain into a Banach space. We can prove that $M(D(\Omega))$ can be identified with $E^{*}$, where $E$ is a separable Banach space and each point evaluation at $\omega \in \Omega$ is a $w e a k^{*}$ continuous linear functional on $M(D(\Omega))$ with norm 1 .

LEMMA 2.2. If $\left(\omega_{n}\right)$ is a sequence in $\Omega_{r}=\{z:|z|<r\}, r<1$, such that $\left|\omega_{n}\right| \rightarrow r$, then there exists $\varphi_{1} \in M\left(D\left(\Omega_{r}\right)\right)$ such that $\lim _{n \rightarrow \infty} \varphi_{1}\left(\omega_{n}\right)$ does not exist and $\varphi_{1}(0)=0$, Furthermore, if $\left(\omega_{n}\right)$ is a sequence in the annulus $A=\{z \in \mathbb{C}: r<|z|<1\}$ and $\left|\omega_{n}\right| \rightarrow$ $r$ or $\left|\omega_{n}\right| \rightarrow 1$, then there is a $\varphi \in M(D(A))$ such that $\lim _{n \rightarrow \infty} \varphi\left(\omega_{n}\right)$ does not exist.

Proof. By $\left[1\right.$, Proposition 4], there exists $\varphi \in M\left(D\left(\Omega_{r}\right)\right)$ such that $\varphi\left(\omega_{n}\right)=(-1)^{n}$. If we consider $\varphi_{1}(z)=z \varphi(z)$, then, by [5, Lemma 2.4], $\varphi_{1} \in M\left(D\left(\Omega_{r}\right)\right), \varphi_{1}(0)=0$ and $\lim _{n \rightarrow \infty} \varphi_{1}\left(\omega_{n}\right)$ does not exist. 
To prove the second part, suppose that $\left|\omega_{n}\right| \rightarrow r$. Considering $\omega_{n}$ in $A_{1}=(\mathbb{C} \cup$ $\{\infty\}) \backslash \Omega_{r}$, since $A_{1}$ is the conformal image of $\left\{z:|z|<r_{1}\right\}$ for some $r_{1}>0$, there exists $\varphi_{2} \in M\left(D\left(A_{1}\right)\right)$ such that $\lim _{n \rightarrow \infty} \varphi_{2}\left(\omega_{n}\right)$ does not exist and vanish at infinity. If $\varphi_{3} \in$ $M(D(U))$, then $\varphi_{3}$ is analytic in the unit disk $U$. Therefore, $\varphi_{3}\left(\omega_{n}\right)$ is convergent.

Now, we write $\varphi=\varphi_{2}+\varphi_{3}$. Then $\varphi \in M(D(A))$ because $M(D(A))=M(D(U))+$ $M_{0}\left(D\left(A_{1}\right)\right)$, where $M_{0}\left(D\left(A_{1}\right)\right)=H_{0}\left(A_{1}\right) \cap M\left(D\left(A_{1}\right)\right)$, (see [5, Theorem 3.2]). Hence, we find a function $\varphi$ in $M(D(A))$ such that $\lim _{n \rightarrow \infty} \varphi\left(\omega_{n}\right)$ does not exist.

If $\left|\omega_{n}\right| \rightarrow 1$, then there exists $\psi_{1} \in M(D(U))$ such that $\lim _{n \rightarrow \infty} \psi_{1}\left(\omega_{n}\right)$ does not exist. If $\psi_{2} \in M_{0}\left(D\left(A_{1}\right)\right)$, then $\psi_{2}$ is analytic on $A_{1}$. Hence, $\psi_{2}\left(\omega_{n}\right)$ is convergent. Therefore, if $\psi=\psi_{1}+\psi_{2}$, then $\psi$ is in $M(D(A))$ and $\lim _{n \rightarrow \infty} \psi\left(\omega_{n}\right)$ does not exist.

THEOREM 2.3. Let $\left(\omega_{n}\right)$ be a sequence in the circular domain $\Omega=U \backslash\left\{K_{1} \cup K_{2} \cup\right.$ $\left.\cdots \cup K_{N}\right\}$, where $K_{i}=\left\{z:\left|z-\alpha_{i}\right| \leq r_{i}\right\}, \alpha_{i} \in U, i=1,2, \ldots, N$, such that $\left|\omega_{n}\right| \rightarrow 1$ or $\left|\omega_{n}\right| \rightarrow r_{i}, i=1,2, \ldots, N$. Then there is a subsequence of $\left(\omega_{n}\right)$ that is interpolating for $M(D(\Omega))$.

Proof. In either cases $\left|\omega_{n}\right| \rightarrow 1$ or $\left|\omega_{n}\right| \rightarrow r_{i}, i=1,2, \ldots, N$, we can prove, in the same way as in [1, Theorem 1], that if case (i) of Rosenthal-Dor theorem is satisfied, then there exists some subsequence of $\left(\omega_{n}\right)$ that is interpolating for $M(D(\Omega))$. So, it is enough to prove that case (ii) of Theorem 2.1 does not hold.

Let $\left|\omega_{n}\right| \rightarrow r_{j}$ for some $j=1,2, \ldots, N$. As above, there is $\varphi_{j} \in M_{0}\left(D\left(\Omega_{j}\right)\right)$ such that $\lim _{n \rightarrow \infty} \varphi_{j}\left(\omega_{n}\right)$ does not exist. If $\varphi_{0} \in M(D(U))$ and $\varphi_{i} \in M_{0}\left(D\left(\Omega_{i}\right)\right), i=1,2, \ldots, N$, then $\varphi_{0}\left(\omega_{n}\right), \varphi_{i}\left(\omega_{n}\right), i=1,2, \ldots, N, i \neq j$ are convergent. Therefore, if $\varphi=\varphi_{0}+\varphi_{1}+$ $\cdots+\varphi_{N}$, then $\varphi \in M(D(\Omega))$ and $\lim _{n \rightarrow \infty} \varphi\left(\omega_{n}\right)$ does not exist because

$$
M(D(\Omega))=M(D(U))+M_{0}\left(D\left(\Omega_{1}\right)\right)+\cdots+M_{0}\left(D\left(\Omega_{N}\right)\right) .
$$

If $\left|\omega_{n}\right| \rightarrow 1$, there is an $\psi_{0} \in M(D(U))$ such that $\lim _{n \rightarrow \infty} \psi_{0}\left(\omega_{n}\right)$ does not exist and if $\psi_{i} \in M_{0}\left(D\left(\Omega_{i}\right)\right), i=1,2, \ldots, N$, then $\psi_{i}\left(\omega_{n}\right)$ are convergent. Hence, $\psi=\psi_{0}+\psi_{1}+$ $\psi_{2}+\cdots+\psi_{N}$ is in $M(D(\Omega))$ and $\lim _{n \rightarrow \infty} \psi\left(\omega_{n}\right)$ does not exist. The proof is complete.

3. Interpolation sequence for the space of multipliers. Let $H$ be a Hilbert space whose elements are analytic functions $f: U \rightarrow \mathbb{C}$. Assume that $1 \in H$ and the operator $M_{z}$ of multiplication by $z$ maps $H$ into itself and point evaluations are bounded linear functionals on $H$.

Consider $D_{\alpha},-\infty<\alpha<\infty$, the space of all analytic functions in $U$ with Taylor series $f(z)=\sum_{n=0}^{\infty} a_{n} z^{n}$ such that

$$
\|f\|_{\alpha}^{2}=\sum_{n=0}^{\infty}(n+1)^{\alpha}\left|a_{n}\right|^{2}<\infty .
$$

If $\alpha=-1$, then we have the Bergman space $L_{a}^{2}$ and if $\alpha=0$, we obtain the Hardy space $H^{2}$. But if $\alpha=1$, the space $D_{1}$ is the Dirichlet space. In this space, $M_{z}$ is not a contraction because if $\varphi$ is a nonconstant function on $\bar{U}$, then $\|\varphi\|_{\infty}<\left\|M_{\varphi}\right\|$ (see [1]). For a nice treatment of this space (see [2, 3, 4]).

A function $\varphi$ analytic on $U$ is called a multiplier of $H$ if $\varphi H \subset H$. The set of all 
multipliers of $H$ is denoted by $M(H)$. It is well known that $M(H) \subset H^{\infty}(U)$. We also assume that $M(H)$ is rotation invariant. For an introduction to Hilbert spaces of analytic functions, see Seddighi [12, 13]. Shields and Wallen [15] have proved that if $H$ is a Hilbert space of analytic functions on a set $S$ and if point evaluations are bounded linear functionals on $H$, also if $M_{z}$ maps $H$ into itself and is a contraction, then $\left\|M_{\varphi}\right\|=\|\varphi\|_{\infty}, \varphi \in M(H)$. Also, see Shields [14].

In this section, we assume that $M_{z}$ is polynomially bounded in the sense that there is a constant $C>0$ such that

$$
\left\|M_{p}\right\| \leq C\|p\|_{\infty}
$$

for every polynomial $p$. Now, let $\varphi \in M(H)$. Because $\varphi \in H^{\infty}(U)$, there is a sequence $\left\{p_{n}\right\}$ of polynomials such that $\left\|p_{n}\right\|_{\infty} \leq\|\varphi\|_{\infty}$ and $p_{n}(z) \rightarrow \varphi(z)$ for every $z \in U$. Now, it is easy to show that

$$
\left\langle M_{p_{n}} f, g\right\rangle \longrightarrow\left\langle M_{\varphi} f, g\right\rangle
$$

for every $f, g \in H$. In other words, $M_{p_{n}} \rightarrow M_{\varphi}$ in the weak operator topology (WOT) of $B(H)$. In fact, if $g=k_{\lambda}$ for some $\lambda \in U$, then (3.3) is true. If $g$ is a finite linear combination of the reproducing kernels $k_{\lambda}$, then (3.3) is again true. Because $H=\bigvee\left\{k_{\lambda}\right.$ : $\lambda \in U\}$, we conclude that every $g \in H$ can be approximated by a sequence of finite linear combinations of the $k_{\lambda}, \lambda \in U$. To prove that (3.3) holds for every $g \in H$, we use an approximation process, inequality (3.2), and the fact that (3.3) is true for finite linear combinations. We can also prove that

$$
\left\|M_{\varphi}\right\| \leq C\|\varphi\|_{\infty} .
$$

This follows from (3.3) and the fact that

$$
\left|\left\langle M_{p_{n}} f, g\right\rangle\right| \leq\left\|M_{p_{n}}\right\|\|f\|\|g\| \leq C\left\|p_{n}\right\|_{\infty}\|f\|\|g\| \leq C\|\varphi\|_{\infty}\|f\|\|g\| .
$$

We recall that the space $H(U)$ of holomorphic functions on $U$ is equipped with the topology of uniform convergence on compact sets and because the point evaluations are continuous, the embedding $H \rightarrow H(U)$ is continuous. We also let $H(\bar{U})$ denote the space of functions holomorphic on neighbourhoods of $\bar{U}$, with the inductive limit topology. In fact, a sequence $\left\{f_{k}\right\}$ in $H(\bar{U})$ converges to a function $f$ if and only if all the functions are analytic in some fixed open set $G$ containing $\bar{U}$, with $f_{k} \rightarrow f$ uniformly on compact subsets of $G$. We assume that $H(\bar{U}) \subset M(H)$.

Now, let $\left(\omega_{n}\right)$ be a sequence in $U$ such that $\left|\omega_{n}\right| \rightarrow 1$. We show that there is a subsequence of $\left(\omega_{n}\right)$ which is interpolating for $M(H)$. Therefore, we must show that case (ii) of Rosenthal-Dor theorem does not hold. To prove this, we need the following lemmas.

LEMmA 3.1. Let $\omega \in U$. Then there exists a function $\psi$ analytic on $\bar{U}$ such that $\psi(\omega)=0 ; \psi(1)=1$; and $\|\psi\|_{\infty}=1$. In general, let $\omega_{1}, \omega_{2}, \ldots, \omega_{n} \in U$. Then there exists $\psi$ analytic on $\bar{U}$ such that

$$
\psi\left(\omega_{1}\right)=\psi\left(\omega_{2}\right)=\cdots=\psi\left(\omega_{n}\right)=0, \quad \psi(1)=1, \quad\|\psi\|_{\infty} \leq 1 .
$$


Proof. Let $\varphi_{\omega}(z)=e^{i \theta}(\omega-z)(1-\bar{\omega} z)^{-1}$ be an analytic automorphism of the disc $U$. By a suitable choice of $\theta$, we have $\varphi_{\omega}(\omega)=0$ and $\varphi_{\omega}(1)=1$. For this choice of $\theta$, let $\psi=\varphi_{\omega}$. Then $\psi(\omega)=0, \psi(1)=1,\|\psi\|_{\infty}=1$ and because $\psi \in H(\bar{U})$, we conclude that $\psi$ is a multiplier.

For the second part, note that, for each $\omega_{i}$, there exists $\varphi_{i}$ satisfying $\varphi_{i}\left(\omega_{i}\right)=0$, $\varphi_{i}(1)=1$, and $\left\|\varphi_{i}\right\|_{\infty}=1$. Let $\psi=\varphi_{1} \varphi_{2} \cdots \varphi_{n}$. Then $\psi$ has the desired properties.

Proposition 3.2. Let $\left(\omega_{n}\right)$ be a sequence in $U$ such that $\left|\omega_{n}\right| \rightarrow 1$. Then there exists $\varphi \in M(H)$ such that $\lim _{n \rightarrow \infty} \varphi\left(\omega_{n}\right)$ does not exist.

Proof. First, we prove that if $0<r<1$ and $\omega_{1}, \omega_{2}, \ldots, \omega_{n} \in U$, then there exists a function $\varphi$ analytic on $\bar{U}$ such that

$$
\varphi\left(\omega_{1}\right)=\cdots=\varphi\left(\omega_{n}\right)=1, \quad \varphi(1)=-1, \quad\|\varphi\|_{\infty} \leq \frac{1}{r} .
$$

Consider

$$
d(\omega, z)=\left|\frac{\omega-z}{1-\bar{\omega} z}\right| .
$$

Let $s>0$ be chosen such that $s=d(r,-r)=2 r /\left(1+r^{2}\right)$. Then $d(0, s)=d(r,-r)$, and so there exists an analytic automorphism $h$ of $U$ such that $h(0)=r, h(s)=-r$. If $\psi$ is the function given by Lemma 3.1 and $\varphi=\frac{1}{r} h \circ(s \psi)$, we have $\varphi(1)=\frac{1}{r} h \circ(s \psi)(1)=$ $\frac{1}{r} h(s)=-1$,

$$
\begin{aligned}
\varphi\left(\omega_{1}\right) & =\varphi\left(\omega_{2}\right)=\cdots=\varphi\left(\omega_{n}\right)=\frac{1}{r} h \circ(s \psi)\left(\omega_{1}\right)=\frac{1}{r} h \circ(s \psi)\left(\omega_{2}\right) \\
& =\cdots=\frac{1}{r} h \circ(s \psi)\left(\omega_{n}\right)=\frac{1}{r} h(0)=1 .
\end{aligned}
$$

Because $h$ is analytic on $\bar{U}$ and $\|h\|_{\infty}=1$, we conclude that $\varphi$ is analytic on $\bar{U}$ and $\|\varphi\|_{\infty} \leq 1 / r$. Now, we let $\left(r_{k}\right)$ be a sequence in $(0,1)$ such that $\prod_{k=1}^{\infty} 1 / r_{k}<\infty$ and we find a sequence satisfying conditions (11), (12), and (13) on [1, page 416], however, instead of condition (14), we assume that

$$
\left\|\varphi_{k}\right\|_{\infty} \leq \frac{1}{r_{k}}
$$

Using (3.4), we get

$$
\left\|M_{\varphi_{1} \varphi_{2} \cdots \varphi_{k}}\right\| \leq C\left\|\varphi_{1} \varphi_{2} \cdots \varphi_{k}\right\|_{\infty} \leq C \prod_{i=1}^{k} \frac{1}{r_{i}} .
$$

Hence, $\left\{M_{\varphi_{1} \varphi_{2} \cdots \varphi_{k}}\right\}_{k=1}^{\infty}$ is norm bounded in $M(H)$. Because $M(H)=E^{*}$, where $E$ is a separable Banach space, we conclude that there is $\varphi \in M(H)$ such that some subsequence of $\left\{M_{\varphi_{1} \varphi_{2} \cdots \varphi_{k}}\right\}$ is weak $*$ convergent to $M_{\varphi}$. Now, if $\left(\omega_{n}\right)$ is a sequence in $U$ such that $\left|\omega_{n}\right| \rightarrow 1$, then $\varphi$ satisfies $\varphi\left(\omega_{n_{k}}\right)=(-1)^{k}$ and $\lim _{k \rightarrow \infty} \varphi\left(\omega_{n_{k}}\right)$ does not exist.

Now, let $H$ be a Hilbert space of analytic functions on a circular domain $\Omega$ such that $1 \in H, z H \subset H$ and point evaluations are bounded linear functionals on $H$. Clearly, $M(H) \subset H^{\infty}(\Omega)$. We also assume that $H^{\infty}(\Omega) \subset M(H)$ and $M_{z}$ is polynomially bounded as in (3.2). Since 


$$
H^{\infty}(\Omega)=H^{\infty}(U)+H_{0}^{\infty}\left(\Omega_{1}\right)+\cdots+H_{0}^{\infty}\left(\Omega_{N}\right),
$$

where $H_{0}^{\infty}\left(\Omega_{i}\right)=H^{\infty}\left(\Omega_{i}\right) \cap H_{0}\left(\Omega_{i}\right), i=1,2, \ldots, N$ (see [7, Theorem 3.1]), by the same technique as in Sections 2 and 3, we can prove that if $\left(\omega_{n}\right)$ is a sequence in $\Omega$ such that $\left|\omega_{n}\right| \rightarrow 1$ or $\left|\omega_{n}\right| \rightarrow r_{i}, i=1,2, \ldots, N$, then there is a subsequence of $\left(\omega_{n}\right)$ that is interpolating for $M(H)$.

4. General domains and transformation to the unit disc. Let $M>0$ be a constant. We say that a compact set $K$ in $\mathbb{C}$ is an $M$-spectral set for an operator $T$ if $\sigma(T) \subset K$ and $\|f(T)\| \leq M\|f\|_{K}$ for every rational function $f$ with poles outside $K$. Here, $\|f\|_{K}=$ $\max \{|f(z)|: z \in K\}$. Let $G$ be an arbitrary bounded domain each point of which is a bounded point evaluation for a Hilbert space $\mathscr{H}$ of functions analytic on $G$ which contains the constant functions and admits multiplication by the independent variable $z, M_{z}$, as a bounded operator. We denote such a Hilbert space by $(\mathscr{H}, G)$. Also, we assume that $\sigma\left(M_{z}\right)=\bar{G}$ is an $M$-spectral set for $M_{z}$ and we may assume that $M(\mathscr{H})=$ $H^{\infty}(G)$ (or we may assume that each bounded component $G_{\alpha}$ of $G$ is conformally equivalent to an open ball $B_{\alpha}$ such that if $A_{\alpha}=\mathbb{C} \cup\{\infty\} \backslash G_{\alpha}$, then $H\left(\bar{A}_{\alpha}\right)$ is contained in the space of multipliers on $\mathscr{H})$. However, we make a simpler assumption than this later. The idea is to transform the problem to the unit disc and to simplify the problem this way. Now, we indicate how our problem that concerns the operator of multiplication on $(\mathscr{H}, G)$ can be transformed into a problem concerning an operator acting on a Hilbert space of functions analytic on a subset of $U$.

Suppose that $H$ is a simply connected domain with $G \subset H$. Let $h: U \rightarrow H$ be the Riemann mapping corresponding to $H$ and set $\Omega=h^{-1}(G)$. This map enables us to perform the desired transformation. First, we need our Hilbert space to consist of functions that are analytic on the corresponding subdomain of U. Therefore, we set

$$
\mathscr{H}_{h}=\mathscr{H} \circ h=\{f \circ h: f \in \mathscr{H}\},
$$

with the inner product defined by

$$
\langle f \circ h, g \circ h\rangle=\langle f, g\rangle \quad(f, g \in \mathscr{H}) .
$$

It is easy to see that $\mathscr{H}_{h}$ is a Hilbert space. Furthermore, the functions in $\mathscr{H}_{h}$ are analytic on $\Omega, \Omega \subset U$. Also, note that point evaluations are bounded on $\mathscr{H}_{h}$ and $\mathscr{H}_{h}$ contains the constants. The operator $C: \mathscr{H} \rightarrow \mathscr{H}_{h}$ given by $C f=f \circ h$ is clearly an isomorphism and satisfies $L_{h} C=C M_{z}$, where $L_{h}: \mathscr{H}_{h} \rightarrow \mathscr{H}_{h}$ defined by $L_{h} g=h g, g \in \mathscr{H}_{h}$, is the operator of multiplication by $h$ on $\mathscr{H}_{h}$. We also observe that the map $\varphi \rightarrow \varphi \circ h$ is an isometric isomorphism of $\mathcal{M}(\mathscr{H}) \rightarrow \mathcal{M}\left(\mathscr{H}_{h}\right)$ just as the map $A \rightarrow C A C^{-1}$ of $\mathscr{B}(\mathscr{H}) \rightarrow \mathscr{B}\left(\mathscr{H}_{h}\right)$ is.

To find a simply connected domain $H$ containing $G$ which has some nice properties, we need the following lemma; whose proof is an application of transfinite induction (see Conway [8, Lemma 4.8, page 400]).

LEMMA 4.1. If $K$ is a compact subset of the plane, then there is a countable ordinal $\alpha_{0}$ such that, for every $\alpha<\alpha_{0}$, there is a component $V_{\alpha}$ of $\mathbb{C} \backslash K$ such that

(a) if 0 is the first ordinal, $V_{0}$ is the unbounded component of $\mathbb{C} \backslash K$;

(b) for each ordinal $\alpha, V_{\alpha}^{-} \cap\left[\cup_{\beta<\alpha} V_{\beta}\right]^{-} \neq \varnothing$; 
(c) if $V \neq V_{\alpha}$ is a component of $\mathbb{C} \backslash K$ and for any $\alpha$, then $V^{-} \cap\left[\cup_{\alpha} V_{\alpha}\right]^{-}=\varnothing$.

The enumeration of the components of $\mathbb{C} \backslash K$ in Lemma 4.1 picks out those components that can be chained to the unbounded component. So, if $K$ is finitely connected, the unbounded component is enumerated but there may be no others.

If $K$ is a compact set and $\left\{V_{\alpha}: \alpha<\alpha_{0}\right\}$ are those components of $\mathbb{C} \backslash K$ that are picked out by Lemma 4.1 and $\hat{K}$ is the polynomially convex hull of $K$, then we set

$$
L=L_{\alpha_{0}}=\hat{K} \backslash \cup_{\alpha<\alpha_{0}} V_{\alpha} \cup W,
$$

where $W=\cup_{\beta} V_{\beta}$ is the union of all those $V_{\beta} \neq V_{\alpha}, \alpha<\alpha_{0}$, such that $\overline{\cup V}_{\beta}$ meets the outer boundary of $\hat{K}$.

Now, let $M_{z}$ act on $(\mathscr{H}, G)$ in such a way that $\sigma\left(M_{z}\right)=\bar{G}$ is an $M$-spectral set for $M_{z}$. Set $K=\bar{G}$ in the preceding inductive process and find $L$. It turns out that $L$ is the smallest compact subset of $\mathbb{C}$ that contains $K$ and has $L^{0}$ simply connected.

Now, we set $H=L^{0}$ in our preceding argument and let $h: U \rightarrow L^{0}$ be the Riemann mapping corresponding to $L^{0}$. Setting $\Omega=h^{-1}(G)$, we transform our problem concerning $M_{z}$ on $(\mathscr{H}, G)$ to similar problems concerning $L_{h}$ on $(\mathscr{K}, \Omega)$, where $\mathscr{K}=\mathscr{H}_{h}$. The domain $\Omega$ has several nice properties such as $\Omega \subset U$ and $\partial U \subset \bar{\Omega}$. By Carathéodory theorem, $h$ extends to a homomorphism $h: \bar{U} \rightarrow L$ under which the boundaries correspond to each other.

By [12, Theorem 3.6], we see that $\sigma\left(L_{h}\right)=\bar{G}$ is a spectral set for $L_{h}$. Another interesting property of $\Omega$ is that it contains a boundary annulus, i.e., there is $0<r<1$ such that $\bar{A} \subset \Omega$, where $A=\{z: r<|z|<1\}$. To see this, let $\left\{V_{y}\right\}$ be the bounded components of $\mathbb{C} \backslash \bar{\Omega}$. Then $\overline{\cup V}_{\gamma} \cap \partial U=\varnothing$. Let $r=\operatorname{dist}\left(\overline{\cup V}_{\gamma}, \partial U\right)$ and choose $r<s<1$ such that $B=\{z:|z|<s\}$ contains $\overline{\cup V}_{\gamma}$. Clearly, $A=\{z: s<|z|<1\}$ satisfies $\bar{A} \subset \Omega$.

LEMMA 4.2. Let $\Omega$ be an open connected subset of the open unit disc $U$ with $\partial U \subset \bar{\Omega}$. Suppose that $\left\{V_{\gamma}\right\}$ are the bounded components of $\mathbb{C} \backslash \bar{\Omega}$ and assume that each $V_{\gamma}$ is the conformal image of some open disc with positive radius. Also we assume $H(\bar{\Omega}) \subset M(\mathcal{H})$. Furthermore if $\left\{\omega_{n}\right\}$ is a sequence in $\Omega$ such that $\left|\omega_{n}\right| \rightarrow 1$ or $\left|\omega_{n}\right|$ converges to a point on $\partial V_{\gamma}$ for some $\gamma$ then there is $\varphi \in \mathcal{M}(\mathscr{H})$ such that $\lim _{n \rightarrow \infty} \varphi\left(\omega_{n}\right)$ does not exist.

Proof. Suppose $\left|\omega_{n}\right| \rightarrow 1$. We must show that if $0<r<1$ and $\omega_{1}, \omega_{2}, \ldots, \omega_{n} \in U$, then there exists a function $\varphi$ in $H(\bar{U})$ such that

$$
\varphi\left(\omega_{1}\right)=\cdots=\varphi\left(\omega_{n}\right)=1, \quad \varphi(1)=-1, \quad\|\varphi\| \leq \frac{1}{r} .
$$

Repeating the argument of Proposition 3.2, we conclude that if $\left\{r_{k}\right\}$ is a sequence in $(0,1)$ such that $\prod_{k=1}^{\infty} 1 / r_{k}<\infty$, then all the conditions for finding a multiplier $\varphi$ such that $\lim _{n \rightarrow \infty} \varphi\left(\omega_{n}\right)$ does not exist hold provided a suitable form of (4) is in effect.

Now, let $\varphi \in H(\bar{U})$. Because $\Omega \subset U$ and $\sigma\left(L_{h}\right)=\bar{G}$ is an $M$-spectral set, we have

$$
\left\|\varphi \circ h^{-1}\left(L_{h}\right)\right\| \leq M\left\|\varphi \circ h^{-1}\right\|_{\bar{G}}=M\|\varphi\|_{\bar{\Omega}}=M\|\varphi\|_{\infty} .
$$

Since $\varphi \circ h^{-1}\left(L_{h}\right)=L_{\varphi}$, we have $\left\|L_{\varphi}\right\| \leq M\|\varphi\|_{\infty}$. Hence,

$$
\left\|L_{\varphi_{1} \varphi_{2} \cdots \varphi_{k}}\right\| \leq M\left\|\varphi_{1} \varphi_{2} \cdots \varphi_{k}\right\|_{\infty} \leq M\left(\prod_{i=1}^{k} \frac{1}{r_{i}}\right) .
$$


Because $\mathcal{M}\left(\mathscr{H}_{h}\right)$ is the dual of a separable Banach space, we conclude that there is $\varphi \in$ $\mathcal{M}\left(\mathscr{H}_{h}\right)$ such that $\left\{\varphi_{1} \varphi_{2} \cdots \varphi_{k}\right\}$ has a subsequence that converges pointwise on $\Omega$ to $\varphi$. Because $\varphi_{p}\left(\omega_{n_{k}}\right)=1$ whenever $p \geq k$, we have $\varphi\left(\omega_{n_{k}}\right)=\lim _{p \rightarrow \infty}\left(\varphi_{1} \cdots \varphi_{p}\right)\left(\omega_{n_{k}}\right)=$ $\left(\varphi_{1} \cdots \varphi_{k-1}\right)\left(\omega_{n_{k}}\right) \cong(-1)^{k-1}$. This shows that $\lim _{k \rightarrow \infty} \varphi\left(\omega_{n_{k}}\right)$ does not exist.

Now suppose that $\left\{\omega_{n}\right\}$ is a sequence such that $\left|\omega_{n}\right|$ converges to a point on $\partial V_{\gamma}$ for some $\gamma$. Fix $\gamma$. Let $\varphi_{0}: V_{\gamma} \rightarrow B(\alpha, r)$ be a conformal mapping, where $\alpha \in \mathbb{C}$ and $r>0$. Let $\varphi_{1}(z)=r /(z-\alpha)$. Then $\varphi_{2}=\varphi_{1} \circ \varphi_{0}$ maps $V_{\gamma}$ onto $U$. Because $\left|\omega_{n}\right|$ converges to a point on $\partial V_{\gamma}$, we conclude that $\left|\varphi_{2}\left(\omega_{n}\right)\right| \rightarrow 1$ and we work with this sequence as above.

ACKNOWLEDGEMENT. The research of the second author is partially supported by a grant from the Shiraz University Research Council.

\section{REFERENCES}

[1] S. Axler, Interpolation by multipliers of the Dirichlet space, Quart. J. Math. Oxford Ser. (2) 43 (1992), no. 172, 409-419. MR 93k:30079. Zbl 768.30023.

[2] S. Axler and A. L. Shields, Univalent multipliers of the Dirichlet space, Michigan Math. J. 32 (1985), no. 1, 65-80. MR 86c:30043. Zbl 567.30036.

[3] L. Brown and A. L. Shields, Cyclic vectors in the Dirichlet space, Trans. Amer. Math. Soc. 285 (1984), no. 1, 269-303. MR 86d:30079. Zbl 537.30040.

[4] L. Carleson, An interpolation problem for bounded analytic functions, Amer. J. Math. 80 (1958), 921-930. MR 22\#8129. Zbl 085.06504.

[5] K. C. Chan, On the Dirichlet space for finitely connected regions, Trans. Amer. Math. Soc. 319 (1990), no. 2, 711-728. MR 90j:46029. Zbl 727.46011.

[6] K. C. Chan and A. L. Shields, Zero sets, interpolating sequences, and cyclic vectors for Dirichlet spaces, Michigan Math. J. 39 (1992), no. 2, 289-307. MR 94d:46025. Zbl 760.30020.

[7] B. Chevreau, C. M. Pearcy, and A. L. Shields, Finitely connected domains G, representations of $H^{\infty}(G)$, and invariant subspaces, J. Operator Theory 6 (1981), no. 2, 375-405. MR 83j:47004. Zbl 525.47004.

[8] J. B. Conway, Subnormal Operators, Pitman (Advanced Publishing Program), Boston, Mass.-London, 1981. MR 83i:47030. Zbl 474.47013.

[9] L. E. Dor, On sequences spanning a complex $l_{1}$ space, Proc. Amer. Math. Soc. 47 (1975), 515-516. MR 50\#10774. Zbl 296.46014.

[10] P. R. Halmos, A Hilbert Space Problem Book, 2nd ed., Springer-Verlag, New York, 1982. MR 84e:47001. Zbl 496.47001.

[11] H. P. Rosenthal, A characterization of Banach spaces containing $l^{1}$, Proc. Nat. Acad. Sci. U.S.A. 71 (1974), 2411-2413. MR 50\#10773. Zbl 297.46013.

[12] K. Seddighi, von Neumann operators in $\Re_{1}(\Omega)$, Canad. Math. Bull. 27 (1984), no. 2, 146156. MR 85i:47019. Zbl 533.47017.

[13] _ _ Reproducing kernel Hilbert spaces, Iranian J. Sci. Tech. 17 (1993), no. 3, 171-177. MR 96e:46036.

[14] A. L. Shields, Weighted shift operators and analytic function theory, Amer. Math. Soc. (1974), 49-128. Math. Surveys, No. 13. MR 50\#14341. Zbl 303.47021.

[15] A. L. Shields and L. J. Wallen, The commutants of certain Hilbert space operators, Indiana Univ. Math. J. 20 (1970/1971), 777-788. MR 44\#4558. Zbl 207.13801.

Mosaleheh: DePartment of Mathematics, Shiraz University, SHIRAZ, IrAN

E-mail address: mosaleheh@math. susc. ac. ir

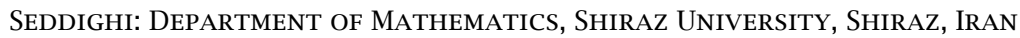




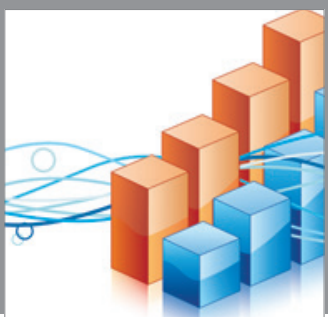

Advances in

Operations Research

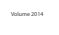

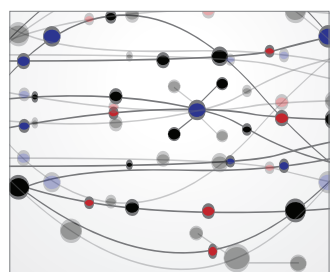

\section{The Scientific} World Journal
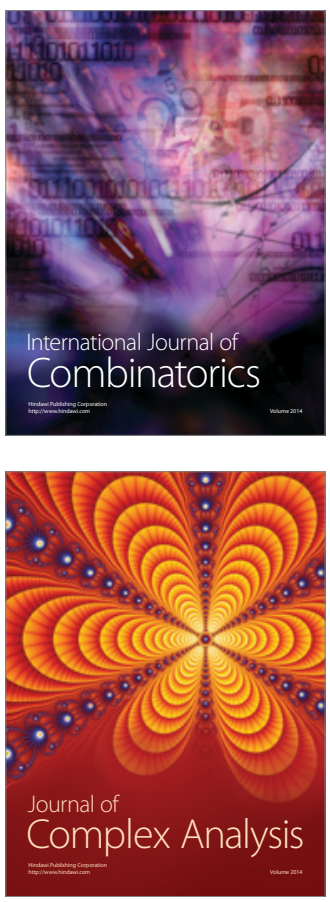

International Journal of

Mathematics and

Mathematical

Sciences
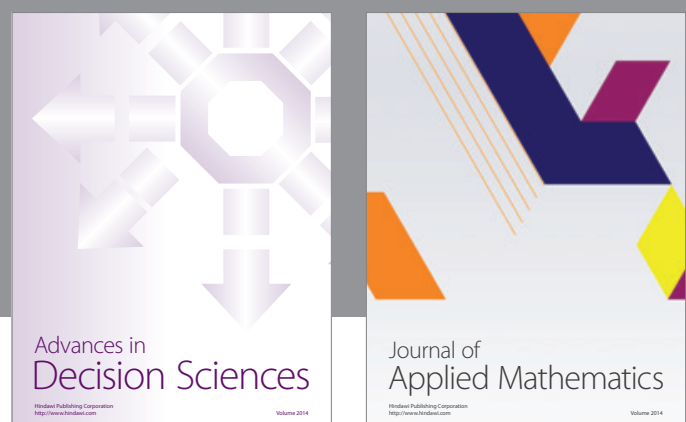

Journal of

Applied Mathematics
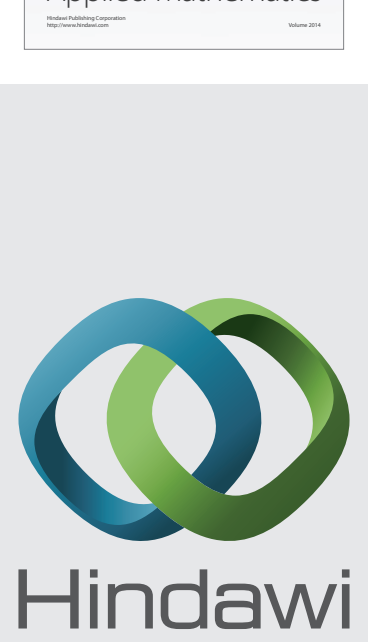

Submit your manuscripts at http://www.hindawi.com
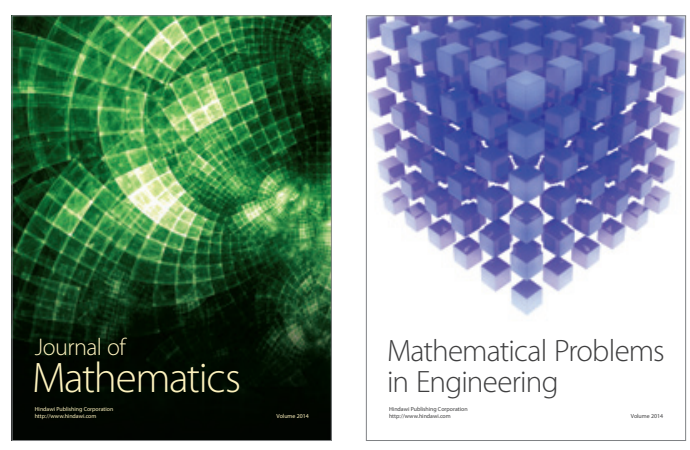

Mathematical Problems in Engineering
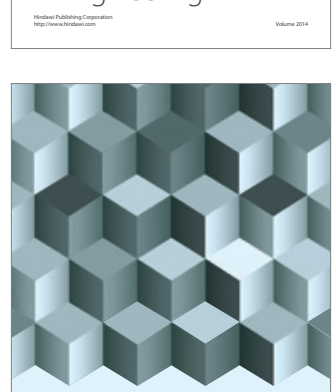

Journal of

Function Spaces
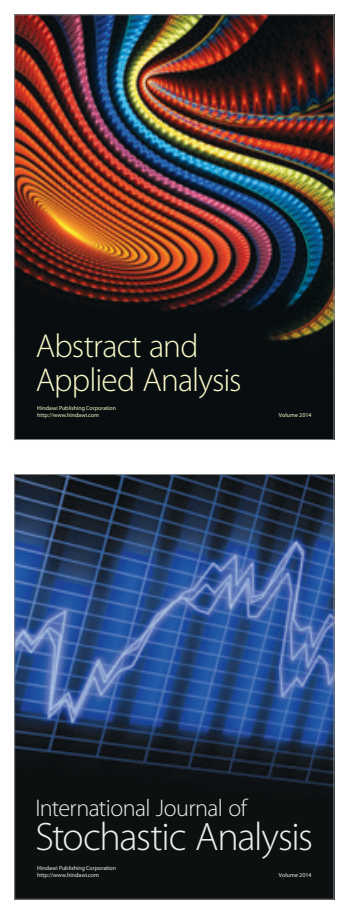

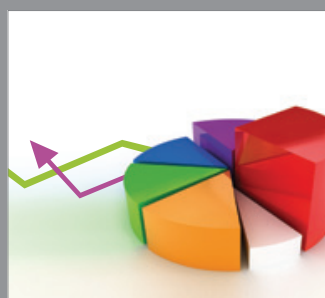

ournal of

Probability and Statistics

Promensencen
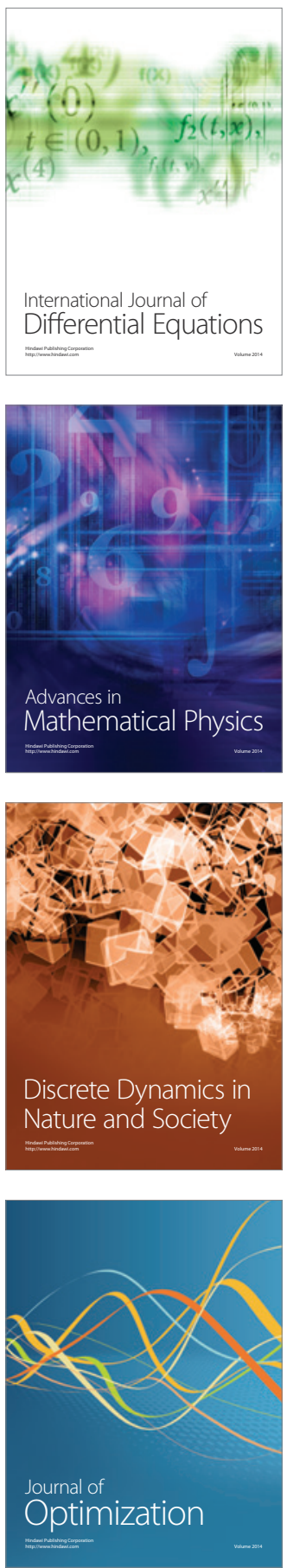